\title{
Tubercular round pneumonia simulating a mass lesion in an adult Ved Prakash ${ }^{a}$, Ajay K. Verma ${ }^{a}$, Ankit Bhatia ${ }^{b}$, Arpita Singh ${ }^{c}$, Surya Kant ${ }^{b}$, Abhijeet Singh $^{\mathrm{a}}$
}

\section{Departments of apulmonary and Critical Care Medicine, ${ }^{b}$ Respiratory Medicine, King George's Medical University, 'Department of Pharmacology, Dr Ram Manohar Lohia Institute of Medical Sciences, Lucknow, Uttar Pradesh, India}

Correspondence to Ajay K. Verma, Department of Pulmonary and Critical Care Medicine, King George's Medical University, Uttar Pradesh, Lucknow, India

Tel: +91991978 8862

e-mail: drajay21@gmail.com

Received 28 May 2018

Accepted 9 July 2018

The Egyptian Journal of Internal Medicine 2018, 30:170-172

\begin{abstract}
Round pneumonia, a benign cause of spherical lesions seen on chest imaging, can many a times be difficult to distinguish from bronchogenic carcinomas. Although relatively less common in adults, this entity is seen in most pulmonary medicine and radiology practices and may lead to computed tomography and biopsy. Because round pneumonia can be easily managed with antibiotics and antitubercular drugs, this diagnosis should be considered in all patients with a spherical lesion, keeping in mind that bronchogenic carcinoma can be a diagnosis as well.
\end{abstract}

\section{Keywords:}

bronchogenic carcinoma, round pneumonia, tuberculosis

Egypt J Intern Med 30:170-172

(1) 2017 The Egyptian Journal of Internal Medicine

1110-7782

\section{Introduction}

Round pneumonia has been defined as an oval or roundshaped consolidation distributed in a nonsegmental pattern, found mostly in children. Owing to its radiological appearances, it stimulates bronchogenic carcinoma particularly in adults. We present a case of tubercular round pneumonia mimicking malignancy, treated successfully by antitubercular drugs.

\section{Case history}

A 40-year-old nonsmoker, nondiabetic male patient was referred to us as a case of nonresolving pneumonia with an antecedent 2 months history of right-sided chest pain and fever. The available chest radiography (Fig. 1) was suggestive of a circumscribed consolidation over the right lower zone. His medical history had been uneventful. Viral markers were nonreactive. Total leukocyte counts were normal $\left(7700 / \mathrm{cm}^{3}\right)$. Patient was subject to repeat sputum examinations for gram staining and acid-fast bacilli staining, both of which could not help with the diagnosis. Computed tomography (CT) scan (Figs 2 and 3) was ordered which revealed a well-defined thick-walled lesion $(9 \times 8.9 \times 8.1 \mathrm{~cm}$ in size $)$ involving lower lobe of right lung with air specks. In view of the mass-like picture, a CT-guided transthoracic fine-needle aspiration cytology/aspirate/biopsy was done. Biopsy of the lung mass revealed granulomatous inflammation consistent with tubercular etiology. The patient was started on standard four-drug regimen of rifampicin, isoniazid, ethambutol, and pyrazinamide according to weight. Follow-up was done using serial chest radiographies every month. A favorable response was seen, as chest pain and fever improved. Treatment was extended to 12 months on stopping pyrazinamide after 3 months. After 12 months of therapy, a follow-up chest radiography (Fig. 4) and CT scan (Figs 5 and 6) showed that the process had resolved. There was near-complete resolution of the rounded mass on imaging studies. Patient was asymptomatic and gained weight and appetite. Anti tubercular therapy (ATT) was stopped, and patient was kept on routine follow-up.

\section{Figure 1}

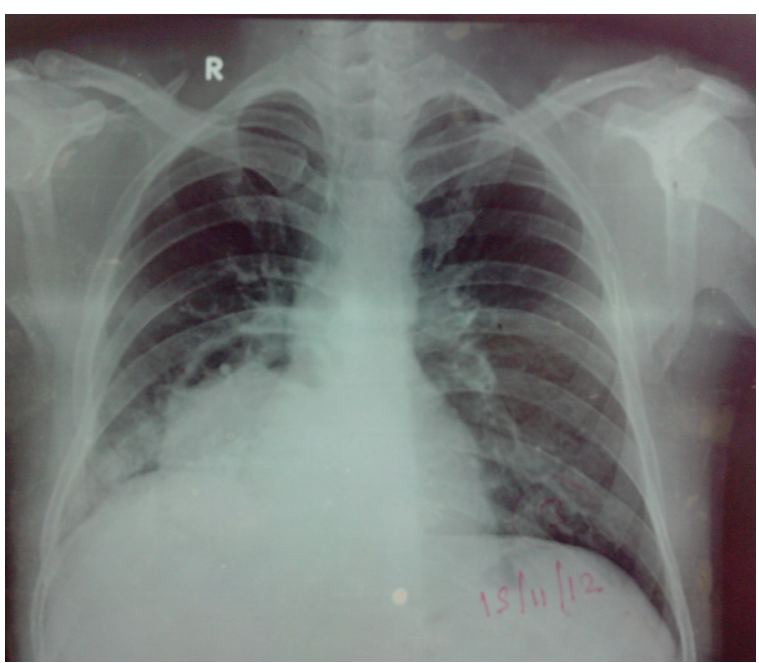

Circumscribed consolidation over right lower zone.

This is an open access journal, and articles are distributed under the terms of the Creative Commons Attribution-NonCommercial-ShareAlike 4.0 License, which allows others to remix, tweak, and build upon the work non-commercially, as long as appropriate credit is given and the new creations are licensed under the identical terms. 
Figure 2

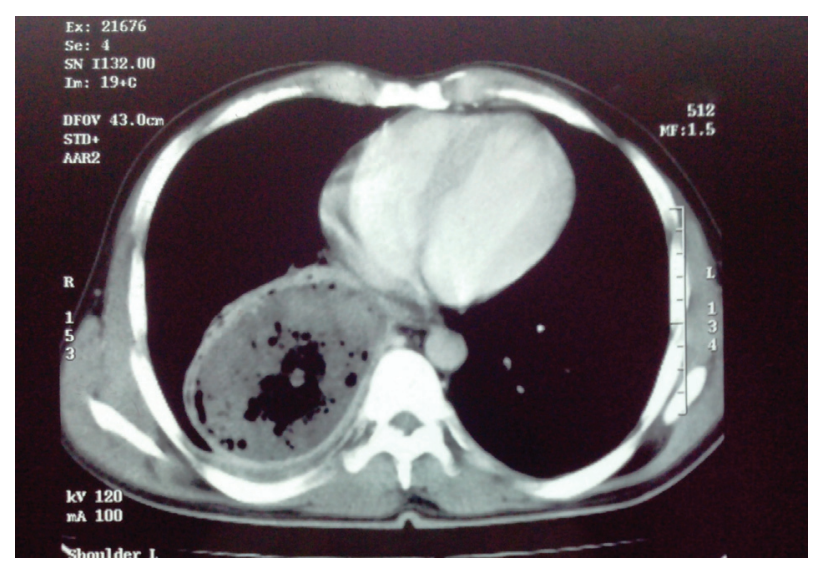

A well defined thick walled lesion $(9 \times 8.9 \times 8.1 \mathrm{~cm})$ involving lower lobe of right lung with air specks.

\section{Figure 3}

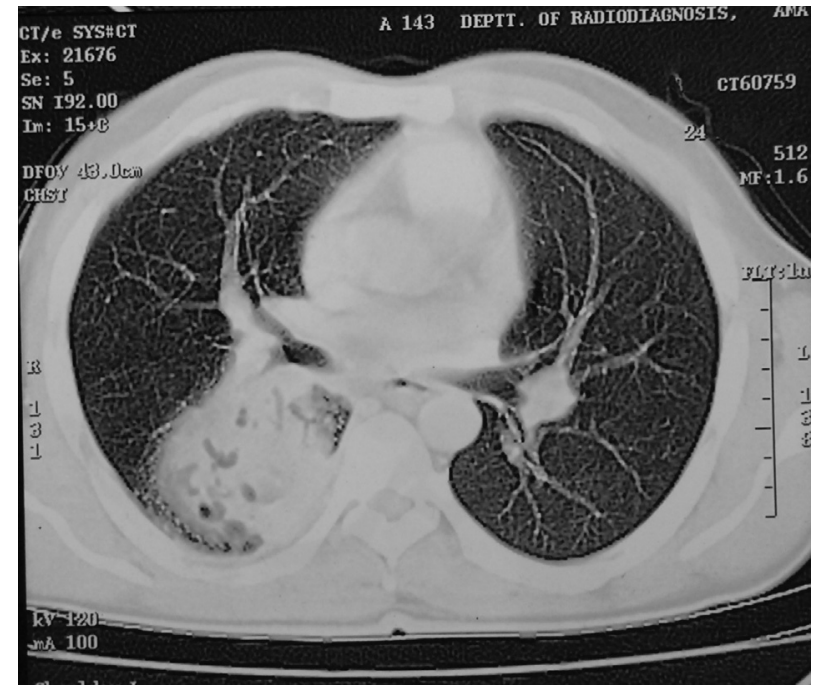

A well defined circumscribed involving lower lobe of right lung.

\section{Figure 4}

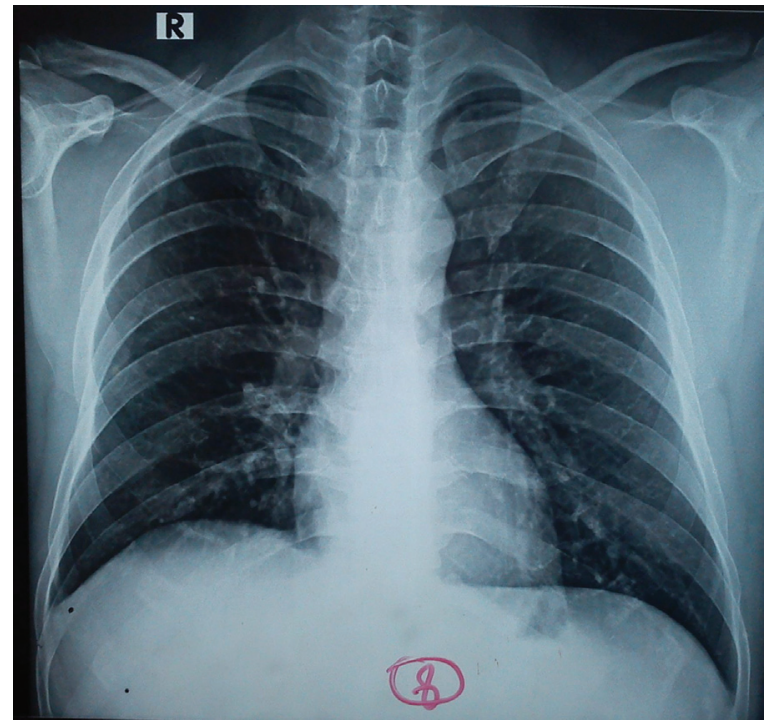

Follow up Chest X-ray showing complete resolution of lesion.
Figure 5

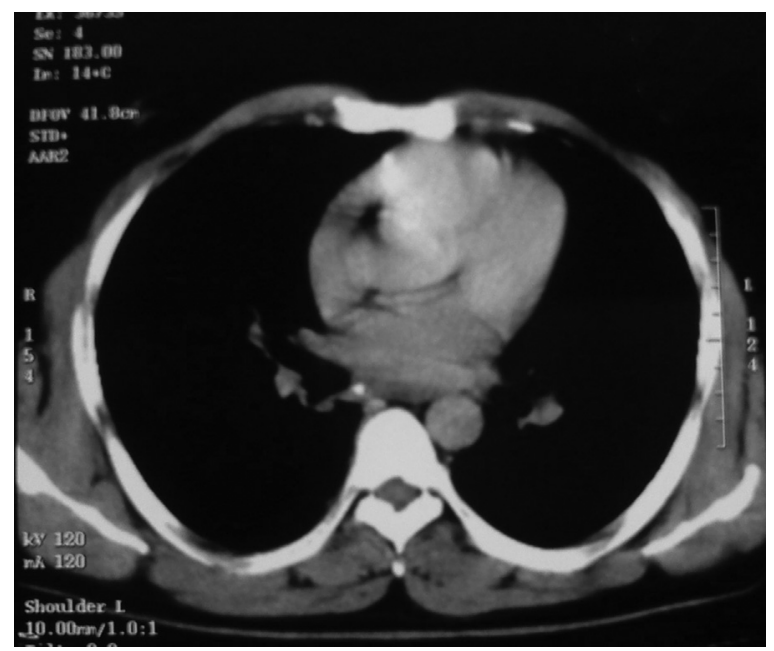

Follow up CECT Thorax showing complete resolution of lesion (Mediastinal Window).

Figure 6

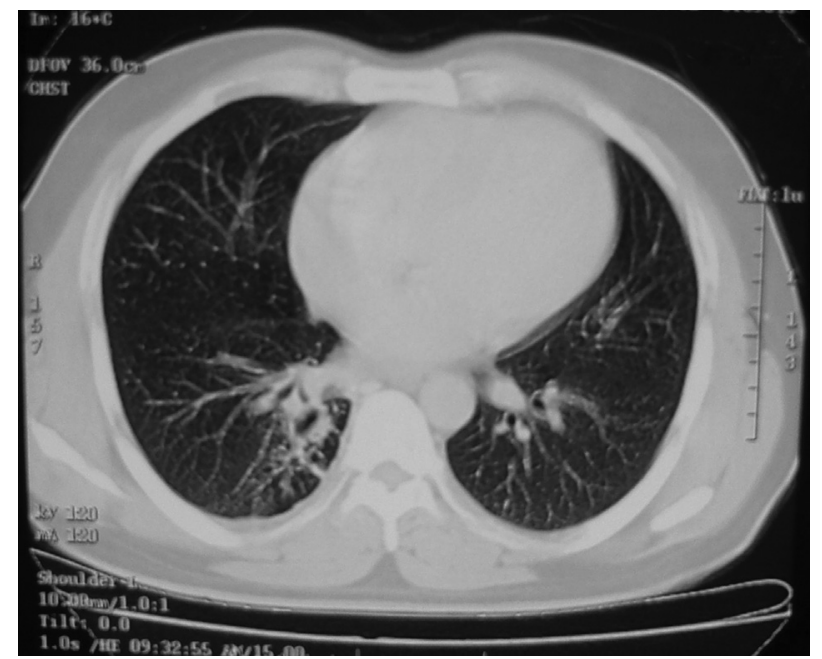

Follow up CECT Thorax showing complete resolution of lesion (Lung Window).

\section{Discussion}

Round pneumonia is a condition usually seen in children, with very few reports recorded in adult patients. It is a type of acute infective pneumonia with a round-shaped consolidation area radiologically [1]. It simulates pulmonary masses both clinically and radiologically. Round pneumonia can be difficult to distinguish from bronchogenic carcinoma particularly in adults. Round pneumonias are roundish and although they are wellcircumscribed parenchymal opacities, they tend to have irregular margins. The typical location is the posterior and lower lobe [2]. Streptococcus pneumonia is the most common pathogen of spherical pneumonia in both adults and children, and other pathogens include Klebsiella pneumoniae, Haemophilus influenzae, and 
Mycobacterium tuberculosis [3]. Unusual radiographic patterns in pulmonary tuberculosis presenting with mass-like densities, most of which were initially and mistakenly diagnosed as neoplasm, have been uncommonly seen in the past [4]. Round pneumonia should be suspected in an adult patient who presents with a pulmonary mass, especially if he has respiratory infection symptoms, is a young nonsmoker, and has no other findings to suggest malignancy. Any patient with a pulmonary nodule that does not decrease in size or resolution after antibiotic treatment should be further assessed with bronchoscopyor transthoracicneedle biopsy.

Tuberculosis continues to be a major cause of morbidity and mortality worldwide. There has been a steady rise in the number of tubercular patients with radiological and clinical characteristics stimulating malignancy accentuating the problem of accurate diagnosis of tuberculosis and malignancy. There are many similarities between both diseases; both are highly prevalent, involve lung parenchyma, and are characterized by similar symptoms. Symptoms such as fever, cough, expectoration, hemoptysis, weight loss, and anorexia are common to both tuberculosis and lung cancer. Owing to high prevalence of tuberculosis in India and radiological similarities, many patients with lung cancer initially get wrongly diagnosed and treated for pulmonary tuberculosis, and there appears to be an unacceptable delay during the diagnosis and treatment of lung cancer. When a combination of clinical, laboratory, and imaging findings does not help to exclude malignancy, transthoracic biopsy can to lead to a correct diagnosis. This helps to start appropriate therapy.

\section{Financial support and sponsorship}

Nil.

\section{Conflicts of interest}

There are no conflicts of interest.

\section{References}

1 Rose RW, Ward BH. Spherical pneumonias in children simulating pulmonary and mediastinal masses. Radiology 1973; 106:179-182.

2 Wagner AL, Szabunio M, Hazlett KS, Wagner SG. Radiologic manifestations of round pneumonia in adults. Am J Roentgenol 1999; 172:549-550.

3 Zinkernagel AS, Schaffner A, Himmelman A. Photo quiz. Round pneumonia due to Streptococcus pneumoniae. Clin Infect Dis 2001; 32:1188.

4 Cherian MJ, Dahniya MH, al-Marzouk NF, Abel A, Bader S, Buerki K, Mahdi OZ. Pulmonary tuberculosis presenting as mass lesions and simulating neoplasms in adults. Australas Radiol 1998; 42:303-308. 